\title{
Renal Anomalies Detected on FDG and 68 Ga PSMA PET-CT Imaging in Cancer Patients
}

Pelin Özcan Kara, ${ }^{1 *}$ Zehra Pınar Koç, ${ }^{1}$ Zeynep Selcan Sağlam, ${ }^{2}$ Yüksel Balcı $^{2}$

${ }^{1}$ Mersin University, Faculty of Medicine, Department of Nuclear Medicine, Turkey

${ }^{2}$ Mersin University, Faculty of Medicine, Department of Radiology, Turkey

\begin{abstract}
Renal anomalies may be seen and detected in all age groups, as an insidentally finding. We report the FDG PET/CT and 68 Ga PSMA findings in cancer patients with renal anomalies and situs inversus totalis in three patients.
\end{abstract}

Keywords: Renal anomalies, PET/CT, FDG, 68Ga-PSMA

\section{Images}

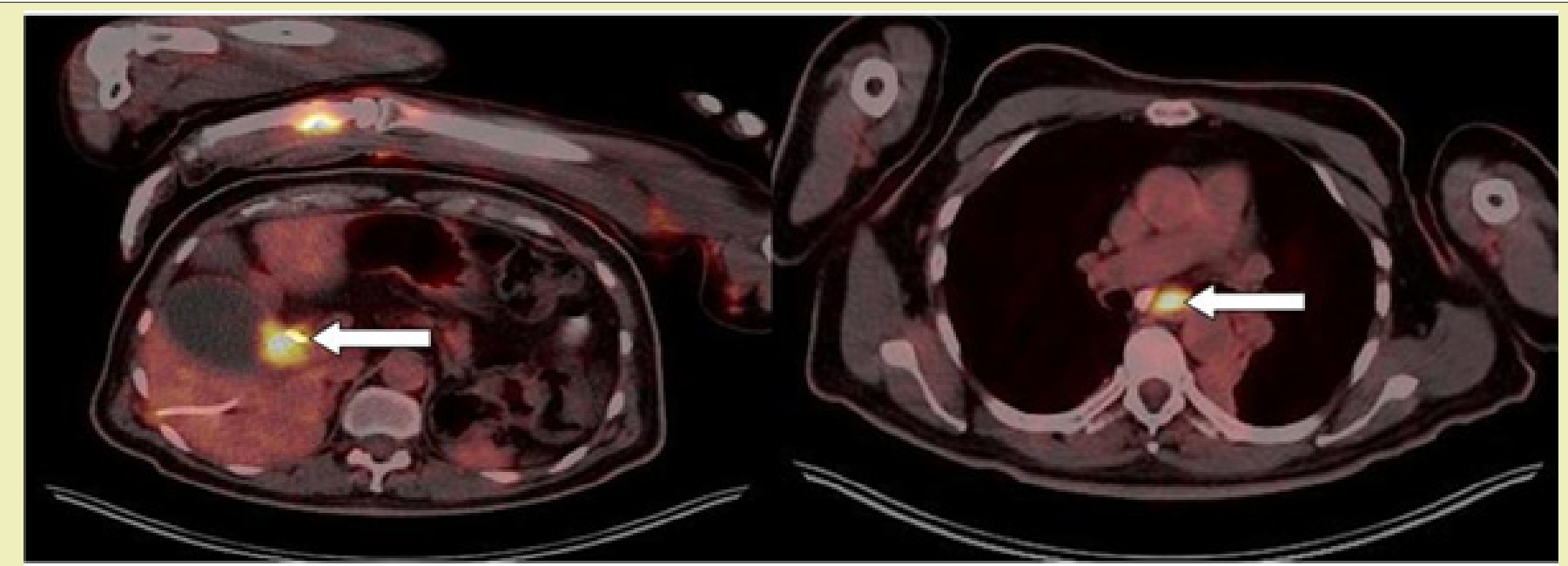

Figure 1: An 65-year-old man with suspicious gastrointestinal system malignancy, underwent PET-CT imaging for metabolic characterization and primary lesion. Following a 4 hour fasting, while the patient had a blood glucose level of $152 \mathrm{mg} / \mathrm{dL}, 303 \mathrm{MBq}(8.2 \mathrm{mCi}) 18 \mathrm{~F}-\mathrm{FDG}$ was given i.v. after $60 \mathrm{~min}$, images were taken from the calvarium to the soles of the feet in 3D mode to be $1 \mathrm{~min}$ per bed. PET-CT imaging demonstrated a subcarinal $1 \mathrm{~cm}$ lymphadenopaty (SUVmax:10.42) and paraosephageal metastatic lympadenopaty (12x22 mm/SUVmax:20.68) at mediastinal region. In addition, an 32x26mm mass compatible with primary malignancy $(32 \times 26 \mathrm{~mm})$ was detected anteromedial section of gallbladder. An approximately $32 \times 26 \mathrm{~mm}$ hypermetabolic mass was detected at pelvic region located cranial and posterior of the bladder. CT component of the PET-CT imaging demonstrated that this mass belonging to a pelvic cake kidney. Both kidneys were not located at normal localisation site. The patient was diagnosed as Klatskin tumor finallay.

Quick Response Code:

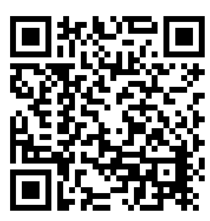

*Corresponding author: Pelin Özcan Kara, Mersin University, Faculty of Medicine, Department of Nuclear Medicine, Turkey

Received: 26 May, 2021

Published: 06 July, 2021

Citation: Kara PO, Koç ZP, Sağlam ZS, Balcı Y. Renal Anomalies Detected on FDG and 68 Ga PSMA PET-CT Imaging in Cancer Patients. Adv Telerad Radiothe. 2021;1(1):1-3. DOI: 10.53902/ATR.2021.01.000501 


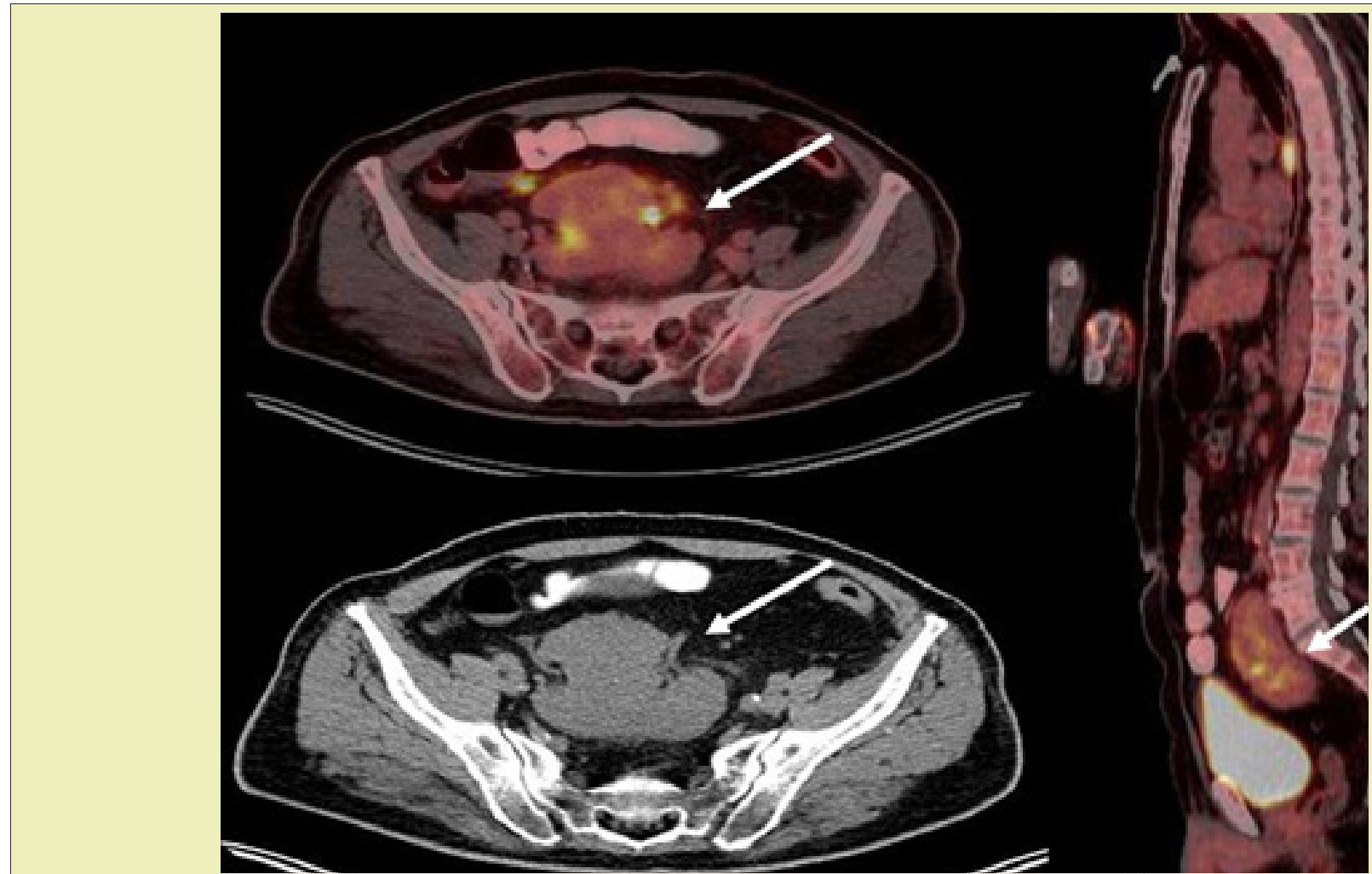

Figure 2: PET-CT imaging normal variants, benign conditions and renal anomalies was reported in literature. ${ }^{1-5}$ In this report, it was noticed that pelvic cake kidney is a rare renal anomaly and may result confusion.

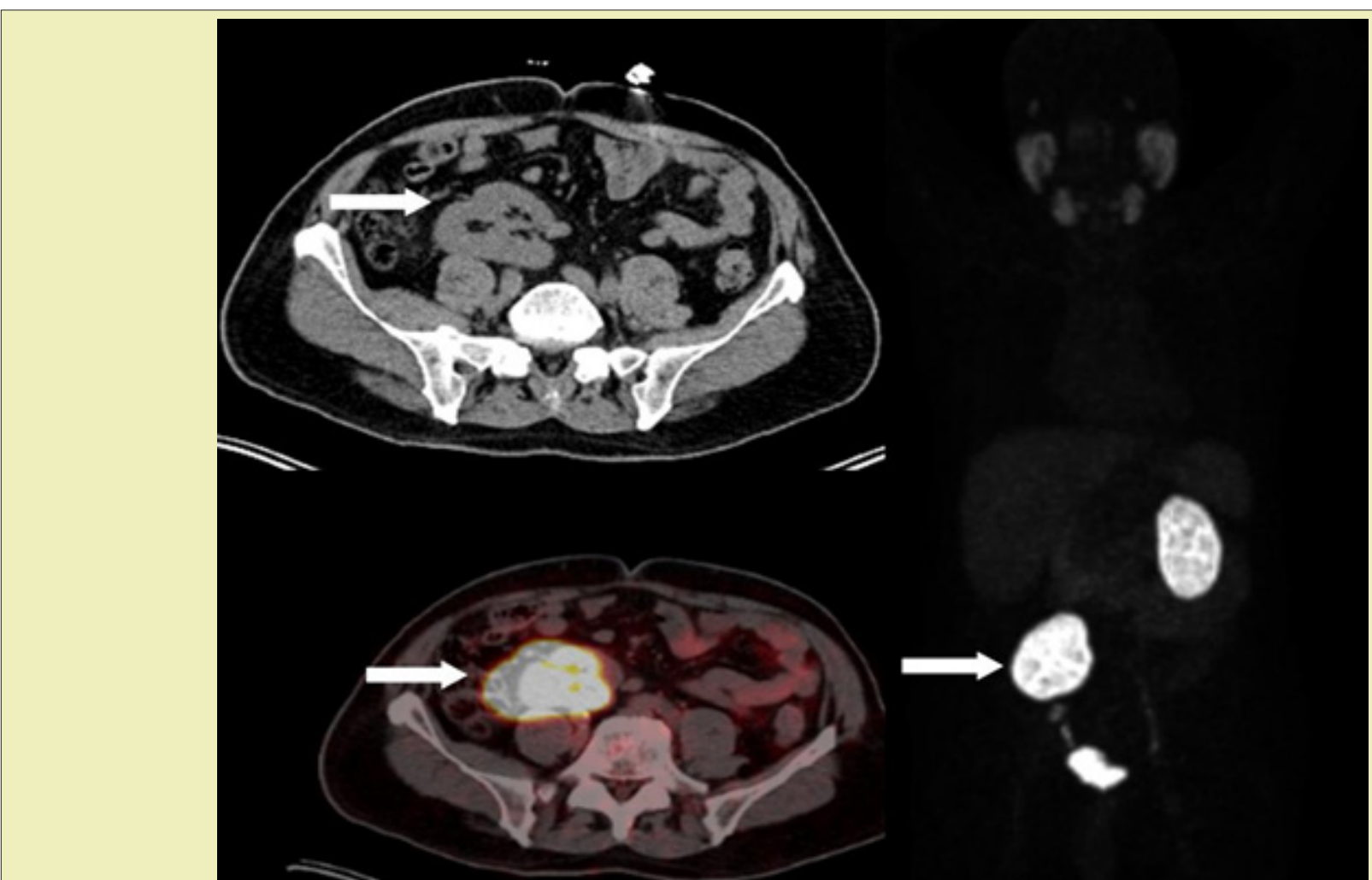

Figure 3: An 62-year-old man with prostate cancer (Gleason skor: 4+5) underwent 68 Ga PET-CT imaging for suspected recurrence. The patient had radical prostatectomy operation history, progresive total PSA level. Total PSA level was $1.2 \mathrm{ng} / \mathrm{ml}(<4.1$ reference level) during imaging. Following iv. injection of $148 \mathrm{MBq}(4 \mathrm{mCi}) 68 \mathrm{Ga}-\mathrm{PSMA}$, images were taken from the calvarium to the upper leg in 3D mode to be $1 \mathrm{~min}$ per bed $60 \mathrm{~min}$. later. PET-CT imaging demonstrated right internal iliak $1.5 \mathrm{~cm}$ metastatic lymph node (SUVmax:26.95) In addition, an ectopic pelvic right kidney was detected with rotation anomaly. CT component of the PET-CT imaging demonstrated that this dense uptake was belonging to a kidney. Left kidney was located at normal localisation site. 

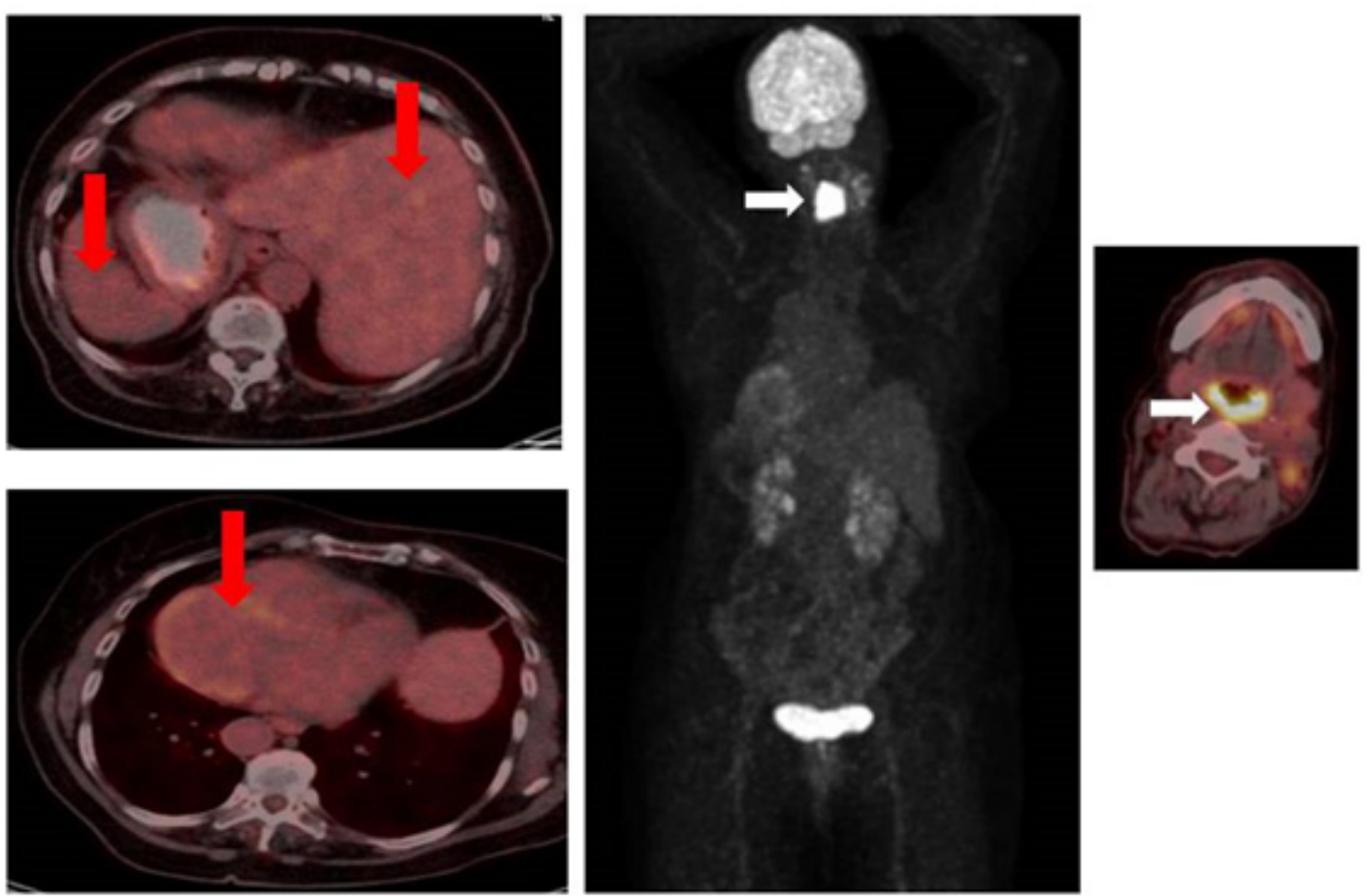

Figure 4: An 63 years of women patient with posterior pharyngeal tumor underwent FDG PET-CT imaging for staging. Primary tumor (SUVmax:23.59) and cervical metastatic lymph nodes (SUVmax:11.84) were demontrates with white arrows. Additionally, the patient had situs inversus totalis anomaly detected insidentally. Myocardium, liver and spleen localisation sites were demonstrated with red arrows.

\section{Acknowledgments}

None.

\section{Funding}

None.

\section{Conflicts of Interest}

Authors declare that there is no conflict of interest.

\section{References}

1. Wang $\mathrm{X}$, Koch S. Positron emission tomography/computed tomography potential pitfalls and artifacts. Curr Probl Diagn Radiol. 2009;38(4):156-169.
2. Shammas A, Lim R, Charron M. Pediatric FDG PET/CT: physiologic uptake, normal variants, and benign conditions. Radiographics. 2009;29(5):1467-1486.

3. Kostakoglu L, Ruth Hardoff R, Rosna Mirtcheva R, et al. PET-CT fusion imaging in differentiating physiologic from pathologic FDG uptake. Radiographics. 2004;24(5):1411-1431.

4. Halac M, Mut SS, Ylmaz S, et al. Appearance of situs inversus totalis and polysplenia syndrome on FDG PET/CT. Clin Nucl Med. 2008;33(2):142-143.

5. Halac M, Yilmaz S, Mut SS, et al. Situs inversus totalis shown in the 18F-FDG PET/CT scan. Hell J Nucl Med. 2007;10(2):121. 